Research Paper

\title{
Severe loss of visceral fat and skeletal muscle after chemotherapy predicts poor prognosis in metastatic gastric cancer patients without gastrectomy
}

\author{
Wanjing Feng, ${ }^{1,2}$, Mingzhu Huang1,2, Xiaoying Zhao',2, Siyuan Chen ${ }^{1,2}$, Chenchen Wang1,2, Jinjia Chang ${ }^{1,2}$, \\ Weijian Guo ${ }^{1,2}$, Zhiyu Chen ${ }^{1,2}$, Hui Zhu ${ }^{2,3}{ }^{\bowtie}$, Xiaodong Zhu ${ }^{1,2}{ }^{\bowtie}$ \\ 1. Department of Medical Oncology, Fudan University Shanghai Cancer Center, 270 Dong' An Road, Shanghai200032, People's Republic of China \\ 2. Department of Oncology, Shanghai Medical College, Fudan University, 130 Dong' An Road, Shanghai 200032, People's Republic of China \\ 3. Department of Radiology, Fudan University Shanghai Cancer Center, 270 Dong' An Road, Shanghai200032, People's Republic of China \\ $\square$ Corresponding authors: Xiaodong Zhu, E-mail: xddr001@163.com or Hui Zhu, E-mail: huizhu171717@163.com \\ () The author(s). This is an open access article distributed under the terms of the Creative Commons Attribution License (https://creativecommons.org/licenses/by/4.0/). \\ See http://ivyspring.com/terms for full terms and conditions.
}

Received: 2019.06.03; Accepted: 2020.02.02; Published: 2020.03.05

\begin{abstract}
Background: The influence of body composition parameters in cancer prognosis attracted researchers' attention. This study investigated the role of visceral fat and skeletal muscle in the prognosis and efficacy of chemotherapy in metastatic gastric cancer (MGC).

Methods: This study included MGC patients without gastrectomy treated with EOF regimen (epirubicin, oxaliplatin and fluorouracil), who participated in a Phase II clinical trial (NCT00767377) with available PACS image data. The visceral fat area (VFA) and skeletal muscle area (SMA) were measured using standard computed tomography (CT).

Results: A total of 46 patients were enrolled in the study. Patients with low baseline VFA and SMA had significantly shorter PFS and OS. In addition, the loss of VFA and SMA also predicts significantly shorter PFS and OS. A prognostic index that included two risk factors, severe loss of VFA and SMA, was used to categorize the patients into two groups: good-risk group (0 risk factors), poor-risk group ( 1 or 2 risk factors). Compared with the good-risk group, the poor-risk group displayed a 3.562-fold-increased risk of progression [hazard ratio $(\mathrm{HR}) 3.652,95 \% \mathrm{Cl} 1.653-7.678 ; \mathrm{P}=0.001$ ] and 2.859-fold-increased risk of death [hazard ratio (HR) 2.859, $95 \% \mathrm{Cl} 1.271-6.434 ; \mathrm{P}=0.011$ ].

Conclusion: Low baseline VFA and SMA, as well as the severe loss of VFA and SMA predict poor prognosis for MGC patients treated by EOF regimen. In patients with severe loss of VFA and/or SMA after 2-cycle chemotherapy, the decision of subsequent chemotherapy should be made after deliberate consideration.
\end{abstract}

Key words: visceral fat, skeletal muscle, metastatic gastric cancer, chemotherapy, prognosis

\section{Introduction}

Gastric cancer (GC) is the fourth most common malignant neoplasm and the second leading cause of cancer-related death worldwide [1]. Unfortunately, the response rate of GC to chemotherapy is low. Even with intensive first-line treatment regimens, such as ECF (epirubicin, cisplatin and fluorouracil), EOF (epirubicin, oxaliplatin and fluorouracil), and EOX (epirubicin, oxaliplatin and capecitabine), the response rate is still $<50 \%$ in patients with metastatic
GC (MGC). Although some patients benefit from chemotherapy, the side effects are unavoidable. Therefore, biomarkers that can predict chemotherapeutic efficacy are urgently needed.

Recently, obesity and sarcopenia are identified as prognostic factor in colorectal cancer $[2,3]$ and hepatocellular carcinoma[4, 5]. Recent evidence suggests that visceral fat plays a key role in carcinogenesis rather than general body fat $[6,7,8,9,10]$. 
Thus, visceral fat might more accurately measure carcinogenesis caused by adipose tissue than body mass index (BMI). Sarcopenia is the progressive and systematic loss of skeletal muscle with atrophy [11] and recognized as a risk factor for physical disability, decreased quality of life and ultimate death.

Therefore, visceral fat area and skeletal muscle area measured by computed tomography (CT) are proposed as body composition factors. CT is a "golden standard" to measure fat [12] and muscle area [13]. These body composition factors have been evaluated in different kinds of tumors [14-17]. In terms of gastric cancer, low visceral fat has been reported as a poor prognostic factor in GC patients with surgery resection [18]. Besides, the loss of visceral fat and skeletal muscle are also reported to predict poor prognostic factor in patients with who underwent gastrectomy [19].

However, no research has investigated whether visceral fat and skeletal muscle can predict prognosis and chemotherapy response in patients with MGC who lose the chance of surgery. The aim of this retrospective study was to reveal the association between body composition parameters measured by computed tomography (CT) and chemotherapy response in patients with MGC.

\section{Materials and Methods}

The current study was a retrospective analysis based on a Phase II clinical trial (NCT00767377) that was conducted at Fudan University Shanghai Cancer Center during June 2007 to July 2012. Due to equipment updates, the new PACS CT system only contains image data after January 2010. Patients with resection of primary lesion and incomplete image data were excluded. All the enrolled patients were confirmed with unresectable MGC and treated with EOF regimen as first-line chemotherapy. Their diagnosis was certified by pathological examination as gastric adenocarcinoma. The specific therapeutic regimen was as described previously [20]. Tumor responses were evaluated in accordance with the Response Evaluation Criteria in Solid Tumors 1.0 (RECIST 1.0) by contrast-enhanced CT scans every two cycles [20].

\section{Measurement of VFA and SMA by CT}

CT images were obtained with multidetector row CT scanners (Somatom Sensation 40 or Somatom Sensation 64; Siemens AG, Medical Solutions, Business Unit CT, Forchheim, Germany), with a slice thickness of $5 \mathrm{~mm}$. Images were uploaded to a picture archiving and communication system (PACS, GE Healthcare-Centricity RIS CE V2.0, GE Medical Systems, US). CT image analysis was performed with the image post-processing software module (AW module) embedded in this PACS. We selected the cross-sectional CT images at the third lumbar vertebra (L3) as a standard landmark to quantify visceral fat area (VFA) and skeletal muscle area(SMA) according to previous literatures $[17,21]$. Two consecutive cross-sectional CT images at L3 level were reformatted by AW module. Preset thresholds of Hounsfield units (HU) were as follows: $-190 \mathrm{HU}$ to $-30 \mathrm{HU}$ for fat tissue, and $-30 \mathrm{HU}$ to $90 \mathrm{HU}$ for skeletal muscle tissue. Total abdominal fat volume (including visceral fat and subcutaneous fat) and skeletal muscle volume of selected two consecutive slices were quantified automatically by AW module. Then, we obtained visceral fat volume by removing subcutaneous fat from total abdominal fat manually. Finally, we could calculate VFA and SMA by dividing these measurements by thickness (two consecutive images, $5 \mathrm{~mm} \times 2=1 \mathrm{~cm}$ ).

\section{Statistical analysis}

Kaplan-Meier survival was used to estimate progression-free survival (PFS) and overall survival (OS) of the four quartiles. Receiver operating characteristic (ROC) curve was used to establish a cut-off value of visceral fat area (VFA) and skeletal muscle area (SMA) to distinguish patients who were expected to have longer PFS and OS from those who were not. Survival curves were estimated by Kaplan-Meier method and analyzed by log-rank test. A multivariate Cox proportional hazards model was used to evaluate the role of VFA and SMA in predicting PFS and OS, after adjusting for clinical characteristics, including age, gender, liver metastasis, lung metastasis, ascites and/or pleural effusion. We use VRV to indicate variation rate of visceral fat area at baseline and after 2-cycle chemotherapy. VRS was used to stand for variation rate of skeletal muscle area at baseline and after two cycles' chemotherapy. Statistical analysis was conducted using Stata version 12.0 software (StataCorp LP, College Station, TX, USA). $\mathrm{P}<0.05$ (two-sided) was considered significant.

\section{Results}

\section{Patient characteristics}

In the new PACS system, image data of baseline and after two-cycle treatment were available only in 53 patients. Among these patients, a total of 46 patients were enrolled in the study after exclusion of patients with gastrectomy. The characteristics of the 46 enrolled patients are shown in Table 1 . The median PFS and OS of the 46 patients were 6.0 months [ $95 \%$ confidence interval (CI) 4.9-7.1] and 19.0 months (95\% CI 10.7-27.3), respectively, which were slightly 
different form the PFS and OS results of the total 150 patients reported previously $(6.0$ months $(95 \%$ CI 5.4-6.6) and 12.6 months (95\% CI 8.2-16.9), respectively) [20].

\section{Baseline VFA/SMA and clinical outcome}

VFA exhibited large variations; therefore, an ROC curve was used to identify a cut-off level to discriminate patients who were expected to have disease progression within a short period $(<6.0$ months) from those who were not. Median PFS in the clinical trial (NCT00767377) was 6.0 months. The area under the curve (AUC) was 0.6004. A VFA value of $62.656 \mathrm{~cm}^{2}$ offered the best overall sensitivity $(76.47 \%)$ and specificity (58.62\%) (Figure 1 ).

Kaplan-Meier survival estimates showed that patients with VFA>62.656 $\mathrm{cm}^{2}$ had significantly longer PFS ( $P=0.003,4.9$ vs. 7.1 months) and OS ( $P=0.039,12.5$ vs. 21.4 months) than those with VFA $\leq$ $62.656 \mathrm{~cm}^{2}$ (Figure 2A, Figure 2B, respectively). In multivariate Cox regression analysis, VFA $>62.656 \mathrm{~cm}^{2}$ was significantly associated with longer PFS (hazard ratio, $0.160 ; 95 \% \mathrm{CI}, 0.048-0.532 ; P=0.003$ ) and OS (hazard ratio, 0.294; 95\% CI, 0.091-0.945; $P=0.040$ ) (Table 2). The objective response rate (ORR) were $60 \%$ in VFA $>62.656 \mathrm{~cm}^{2}$ group and $38 \%$ in $\mathrm{VFA} \leq 62.656$ $\mathrm{cm}^{2}$. ORR was not significantly associated with VFA $(p=0.236)$.
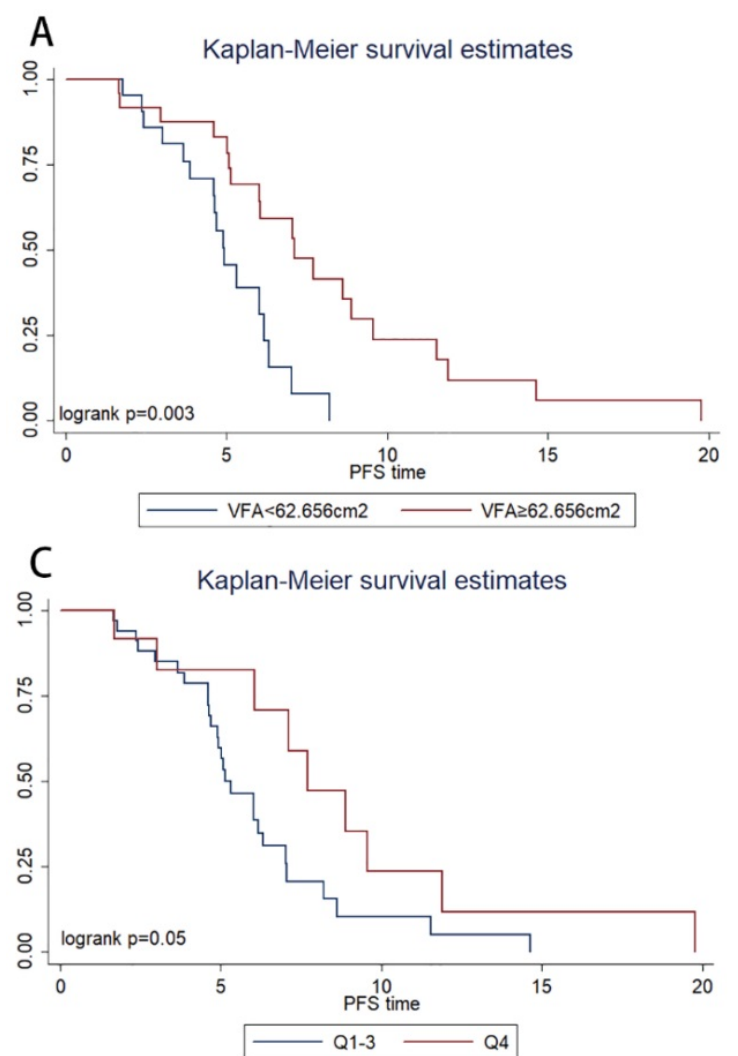

However, an ROC curve failed to identify a cut-off level of SMA to discriminate patients who were expected to have disease progression within a short period ( $<6.0$ months) from those who were not. The patients were categorized into four quartiles (Qs) based on baseline SMA $\left(\mathrm{Q} 1, \leq 99.1 \mathrm{~cm}^{2} ; \mathrm{Q} 2,99.1\right.$ to $118.3 \mathrm{~cm}^{2}$; Q3, 118.3 to $133.9 \mathrm{~cm}^{2}$; and Q4, $\geq 133.9 \mathrm{~cm}^{2}$ ). In the Kaplan-Meier analysis, the high SMA group (Q4) had a significantly longer PFS $(P=0.050$, Figure $2 \mathrm{C})$ and $\mathrm{OS}(P=0.014$, Figure $2 \mathrm{D})$ than the low SMA group (Q1-3). ORR was not significantly associated with SMA $(P=0.738)$.

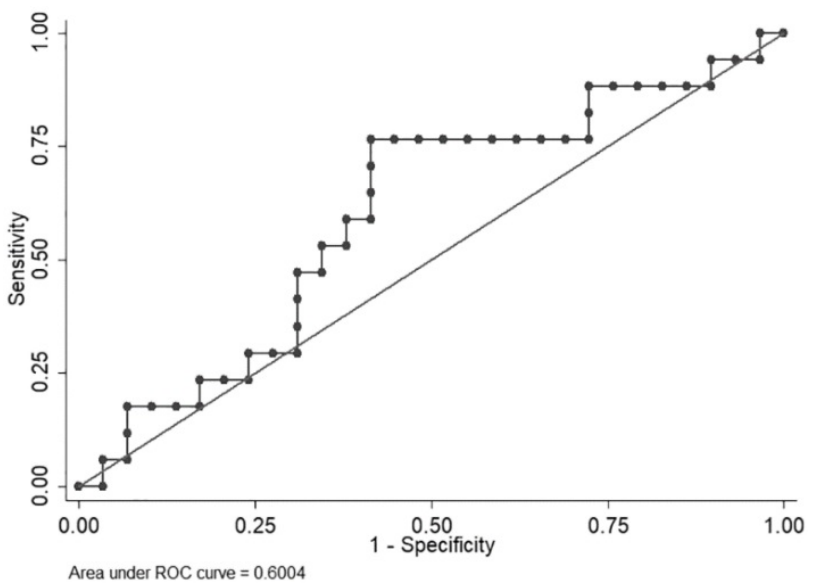

Figure 1. ROC curve of visceral fat area to identify patients with longer progression-free survival time.
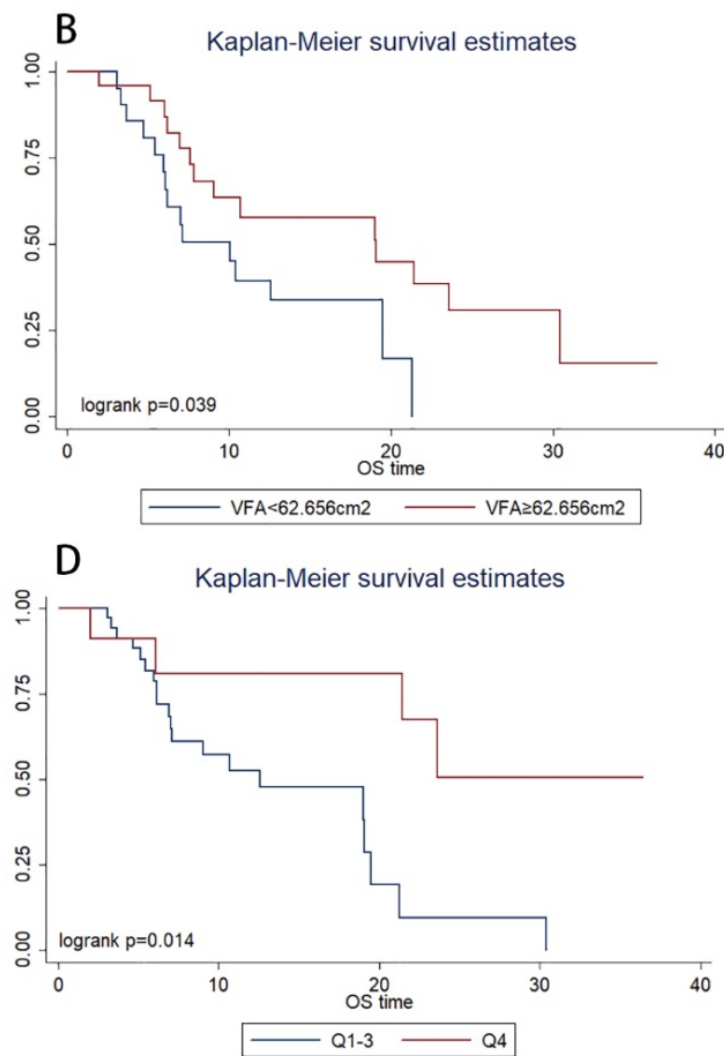

Figure 2. Kaplan-Meier progression-free survival(A) and overall survival(B) curve in patients with different value of baseline visceral fat area (VFA). Kaplan-Meier progression-free survival $(C)$ and overall survival(D) curve in patients with different baseline skeletal muscle area. 
Table 1. Clinicopathologic characteristics of 46 patients.

\begin{tabular}{ll}
\hline Characteristics & Number of Patients \\
\hline Sex & $29(63.0 \%)$ \\
Male & $17(37.0 \%)$ \\
Age (year) & \\
$\geq 60$ & $30(65.2 \%)$ \\
$<60$ & $16(34.8 \%)$ \\
ECOG & \\
0 & $3(6.5 \%)$ \\
1 & $42(91.3 \%)$ \\
2 & $1(2.2 \%)$ \\
Liver Metastasis & \\
Yes & $20(43.5 \%)$ \\
No & $26(56.5 \%)$ \\
Lung Metastasis & \\
Yes & $4(8.7 \%)$ \\
No & $42(91.3 \%)$ \\
retroperitoneal lymph nodes & \\
Yes & $20(43.5 \%)$ \\
No & $26(56.5 \%)$ \\
ascites & \\
Yes & $12(26.1 \%)$ \\
No & $34(73.9 \%)$ \\
hydrothorax & \\
Yes & $5(10.8 \%)$ \\
No & $41(89.2 \%)$ \\
\hline
\end{tabular}

Table 2. Multivariate Cox regression analysis of prognostic factors for progression-free survival and overall survival (visceral fat area as categorized variate).

\begin{tabular}{lllll}
\hline \multirow{2}{*}{$\begin{array}{l}\text { Clinical } \\
\text { characteristics }\end{array}$} & \multicolumn{2}{l}{ Progression-free survival } & Overall survival & \\
\cline { 2 - 5 } & Hazard ratio(95\% CI) & $\mathbf{p}$ & Hazard ratio(95\% CI) & $\mathbf{p}$ \\
\hline Gender & $4.010(0.940-17.117)$ & 0.061 & $0.881(0.184-4.218)$ & 0.874 \\
Age & $1.060(1.001-1.123)$ & 0.046 & $1.027(0.970-1.088)$ & 0.360 \\
Liver metastasis & $0.336(0.140-0.806)$ & 0.015 & $0.318(0.121-0.837)$ & 0.020 \\
Lung metastasis & $0.408(0.066-2.509)$ & 0.333 & $0.822(0.126-5.373)$ & 0.838 \\
Ascites and/or & $0.261(0.087-0.784)$ & 0.017 & $0.274(0.074-1.015)$ & 0.053 \\
pleural effusion & & & & \\
Visceral fat area & $0.160(0.048-0.532)$ & 0.003 & $0.294(0.091-0.945)$ & 0.040 \\
Skeletal muscle area & $1.017(0.991-2.509)$ & 0.199 & $0.999(0.970-1.029)$ & 0.938 \\
\hline
\end{tabular}

Table 3. Multivariate Cox regression analysis of prognostic factors for progression-free survival and overall survival.

\begin{tabular}{|c|c|c|c|c|}
\hline \multirow{2}{*}{$\begin{array}{l}\text { Clinical } \\
\text { characteristics }\end{array}$} & \multicolumn{2}{|c|}{ Progression-free survival } & \multicolumn{2}{|l|}{ Overall survival } \\
\hline & Hazard ratio(95\% CI) & $\mathrm{p}$ & Hazard ratio( $95 \% \mathrm{CI})$ & $\mathrm{p}$ \\
\hline Gender & $2.581(0.998-6.678)$ & 0.051 & $1.119(0.426-2.941)$ & 0.820 \\
\hline Age & $0.549(0.249-1.209)$ & 0.137 & $0.777(0.353-1.709)$ & 0.530 \\
\hline Liver metastasis & $0.398(0.157-1.009)$ & 0.052 & $0.372(0.138-1.003)$ & 0.051 \\
\hline Lung metastasis & $0.666(0.141-3.148)$ & 0.608 & $0.471(0.097-2.296)$ & 0.352 \\
\hline $\begin{array}{l}\text { Ascites and/or } \\
\text { pleural effusion }\end{array}$ & $0.416(0.161-1.076)$ & 0.070 & $0.400(0.133-1.207)$ & 0.104 \\
\hline $\begin{array}{l}\text { Severe loss of VFA } \\
\text { and/or SMA }\end{array}$ & $0.325(0.134-0.788)$ & 0.013 & $0.352(0.137-0.903)$ & 0.030 \\
\hline
\end{tabular}

\section{Severe loss of VFA/SMA and clinical outcome}

The patients were categorized into four quartiles (Qs) based on variation rate of VFA (VRV) (Q1, $\leq$ $-20 \%$; Q2, $-20 \%$ to $0 \%$; Q3; $0 \%$ to $29 \%$; and Q4; >29\%). In the Kaplan-Meier analysis, severe VFA loss group (Q1; patients with visceral fat loss $\geq 20 \%$ ) had a significantly shorter PFS than non-severe VFA loss group (Q2-4; patients with visceral fat loss $<20 \%$ or with visceral fat increase) $(P=0.001$, Figure $3 \mathrm{~A})$ and $\mathrm{OS}$ $(P=0.033$, Figure $3 \mathrm{~B})$. ORR was not significantly associated with VRV $(P=0.491)$.

The patients were categorized into four quartiles (Qs) based on variation rate of SMA (VRS) (Q1, $\leq-8 \%$; Q2, $-8 \%$ to $-3 \%$; Q3; $-3 \%$ to $2 \%$; and Q4; >2\%). In the Kaplan-Meier analysis, severe SMA loss group (Q1; patients with muscle loss $\geq 8 \%$ ) had a significantly shorter PFS ( $P=0.003$, Figure $3 C)$ and OS $(P=0.011$, Figure $3 \mathrm{D}$ ) than non- severe SMA loss group (patients with muscle loss $<8 \%$ or with muscle increase). ORR was not significantly associated with VRS $(P=0.738)$.

\section{Combination analysis of Severe loss of VFA and SMA}

The severe loss of VFA or SMA after chemotherapy was considered as two risk factors and we did a combination analysis of the two factors. Patients were categorized into two groups: the good-risk group $(\mathrm{n}=31)$, patients with no risk factors; and the poor-risk group $(\mathrm{n}=15)$, patients with one or two risk factors. We found that poor-risk group had significantly shorter PFS $(3.8 \mathrm{~m}$ vs $7.0 \mathrm{~m} ; P=0.000$, Figure $4 \mathrm{~A})$ and $O S(7.1 \mathrm{~m}$ vs $19.0 \mathrm{~m} ; P=0.008$, Figure $4 \mathrm{~B})$ than patients in good-risk group. In multivariate Cox regression analysis, severe loss of VFA and/or SMA is an independent predictive factor for shorter PFS (hazard ratio, $0.325 ; 95 \% \mathrm{CI}, 0.134-0.788 ; P=0.013$ ) and OS (hazard ratio, $0.352 ; 95 \% \mathrm{CI}, 0.137-0.903 ; P=0.030$ ) (Table 3). Compared with the good-risk group, the poor-risk group displayed a 3.562-fold-increased risk of progression [hazard ratio (HR) 3.652, 95 \% CI 1.6537.678; $P=0.001$ ] and 2.859-fold-increased risk of death [hazard ratio (HR) 2.859, 95\% CI 1.271-6.434; $P=0.011$ ].

In addition, we performed further analysis in patients with partial response (PR) to chemotherapy (Figure 4C, Figure 4D). In PR patients, whose tumor retraction were favorable to chemotherapy, the median OS of patients with poor risk was only $7.8 \mathrm{~m}$, which was significantly shorter than that of patients with good-risk $(21.4 \mathrm{~m}, P=0.01)$ and shorter than that of the 46 patients $(19.1 \mathrm{~m})$.

\section{Discussion}

We found that low baseline visceral fat area and skeletal muscle area were both significantly associated with shorter PFS and OS in MGC patients treated with EOF regimen. Moreover, the multivariate risk factor model confirmed that VFA was an independent predictive factor for PFS and OS. Baseline VFA and SMA, like Karnofsky score, reflects patients' nutrition status and performance status. Good visceral fat and skeletal muscle reserves indicate that the patients are in good physical condition to receive chemotherapy. 

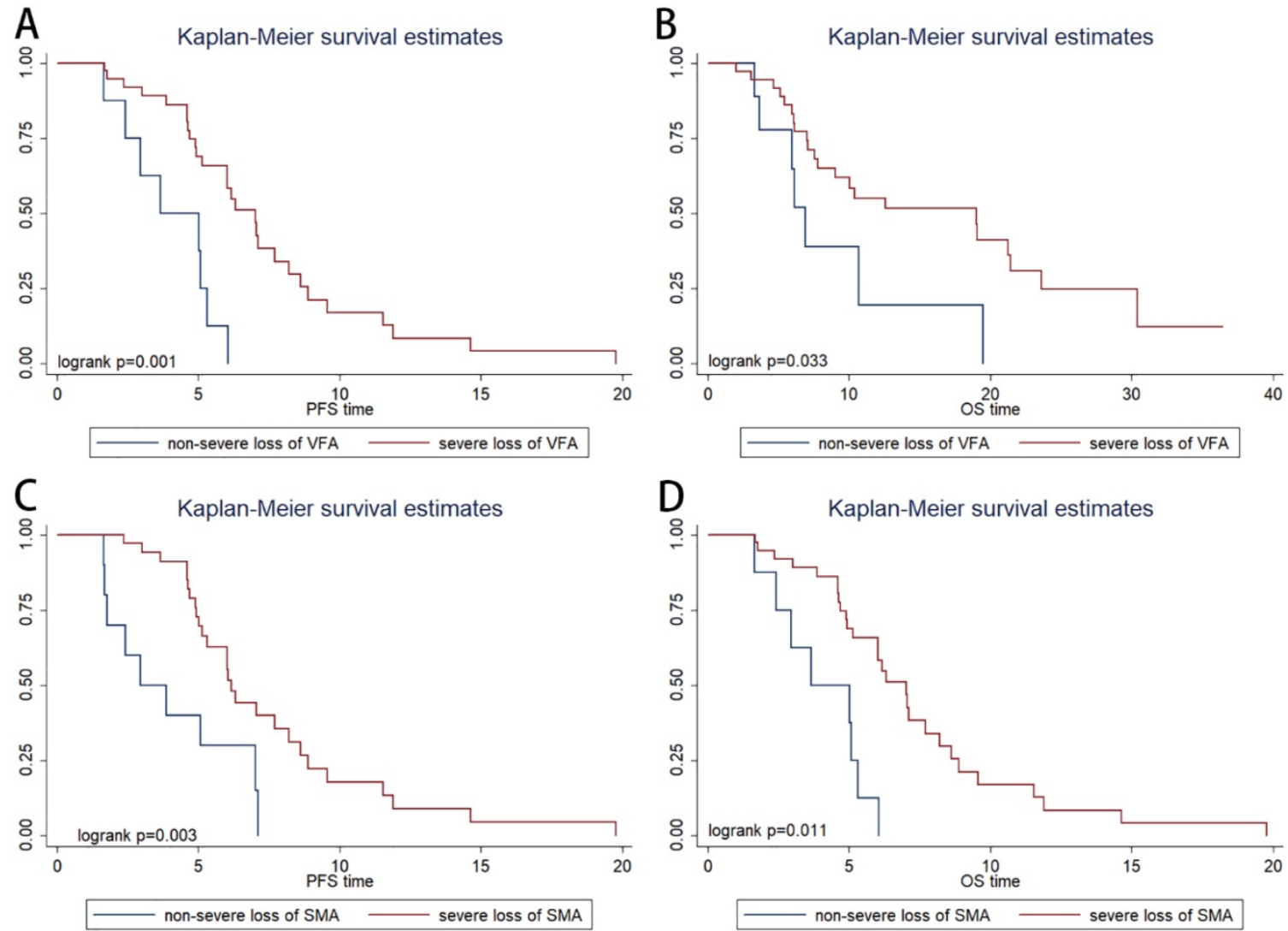

Figure 3. Kaplan-Meier progression-free survival (A) and overall survival (B) curve in patients with different variation rate of visceral fat area. Kaplan-Meier progression-free survival $(C)$ and overall survival $(D)$ curve in patients with different variation rate of skeletal muscle area.
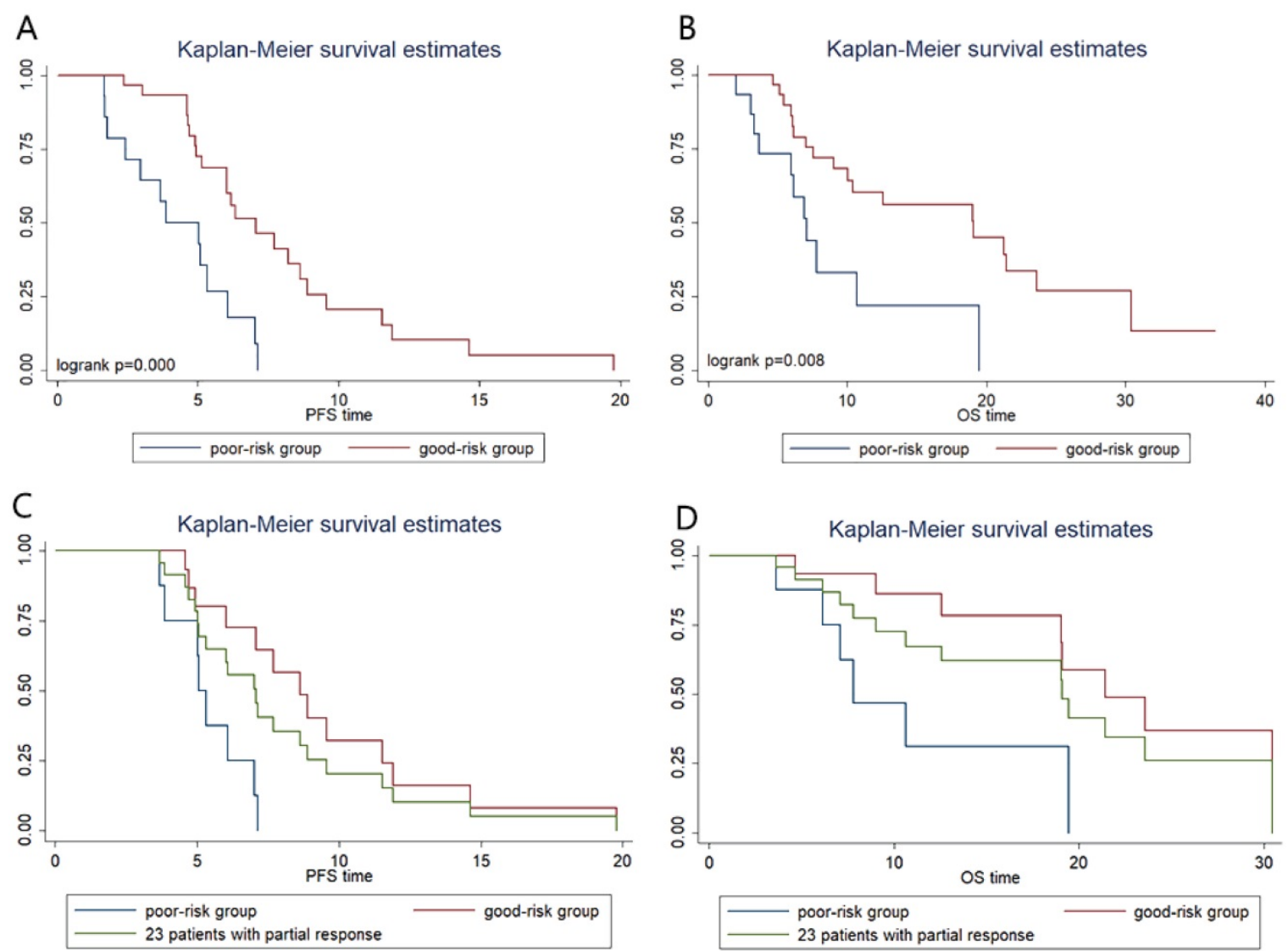

Figure 4. Kaplan-Meier progression-free survival and overall survival curve according to prognostic index. (A) and (B) show differences in PFS and OS, respectively. In patients with partial response, Kaplan-Meier progression-free survival $(C)$ and overall survival (D) curve according to prognostic index. 
We found severe loss of VFA or SMA is associated with shorter PFS and OS. Then we combined the two risk-factors of severe loss of VFA and SMA to create a risk model, and our data confirmed that severe loss of VFA or SMA was an independent predictive factor for PFS and OS. For severe loss of VFA and/or SMA are the individualized responses of each patient to specific chemotherapy regimen, our risk model is helpful in judging patients' tolerance to chemotherapy, trends in disease development, prognosis, and clinical benefit.

One more interesting point of our findings is that we found our risk model is more precise in evaluating further clinical benefit than chemotherapeutic response. It is proven that patients with partial response (PR) to chemotherapy have better PFS and OS than patients with stable disease (SD) or with progression disease (PD) [22]. PR is considered as a good prognostic factor, and patients with $P R$ is always believed to get benefit from chemotherapy. However, our result indicated that even in patients with partial response, the median OS of those with severe loss of VFA or SMA was significantly shorter than that of those without (7.8 months versus 21.4 months). The huge difference suggested that for those patients the survival benefit of further chemotherapy was rather limited. The median number of the courses of chemotherapy in patients with severe loss of VFA/SMA was $6(5-8)$, and the number in patients without severe loss of VFA/SMA was 6(5-8). It means that there is no significant difference in the chemotherapy intensity between the two groups, which exclude the possibility that the difference in survival between the two groups was due to the reduction of subsequent chemotherapy intensity in patients with severe loss of VFA/SMA. Therefore, for those patients, improvement of the poor nutritional status may be more important than chemotherapy. In this situation, the decision of whether patients still need subsequent chemotherapy should be made after deliberate consideration.

These composition parameters, including VFA, SMA, VRV and VRS, can be used together with performance status and tumor biomarkers, and help us to make clinical decisions. In patients with difficulties in evaluating efficacy after two-cycle chemotherapy, these parameters are especially useful for oncologists to evaluate whether patients will benefit from subsequent chemotherapy.

The composition parameters are closely related to malnutrition. Malnutrition may affect the tolerance of chemotherapy, quality of life and survival [23,24]. Cachexia, which is a life-threatening condition and observed in $85 \%$ of gastric cancer patients [25], accounts for more than $20 \%$ of cancer death [26, 27].
Cachexia is composed of the loss of skeletal muscle and adipose tissue. Sarcopenia is observed in different kinds of cancer, which is associated with prognosis. Cancer cells produce inflammatory cytokines, resulting in systematic inflammation. These inflammatory cytokines may trigger muscle wasting and skeletal muscle atrophy, resulting in sarcopenia [26, 28]. The loss of skeletal muscle is also considered to predict poor prognosis in colorectal cancer [14] and gastric cancer [19].

Visceral fat is considered as a poor prognostic factor for survival in colorectal cancer [29-33] and pancreatic cancer [34]. However, in our study, low visceral fat and the loss of visceral fat predicts significant poor prognosis, which is supported by previous studies. Hyung S.P. et al conducted a single-center retrospective study from the CLASSIC Trial and revealed that the marked loss of visceral fat predicts shorter DFS and OS in GC patients who underwent gastrectomy [19]. Another retrospective study conducted by Kazuto H. showed that patients with upper gastrointestinal cancer with low visceral fat content had shorter OS than those with high visceral fat content [18]. We suggest three reasons for the opposite results in gastric cancer that low visceral fat predicts poor prognosis. First, all the enrolled patients are in stage IV, the oral ingestion disorder of which is poorer than patients in early stage. Nutritional deficiency can be caused by decreased intake, resulting in cachexia, which is associated with poor cancer prognosis. Second, fat is an energy reserve and loss of fat is a part of nutritional deficiency. Patients with better nutritional status and energy reserves are expected to live longer. Third, the loss of visceral fat can be considered as the process of energy consumption. The more energy is consumed, the shorter lifetime is expected.

In our study, patients who underwent the resection of primary lesion were excluded and there are two reasons. First, gastrectomy might remove the visceral adipose tissue, which affects the value of baseline visceral fat area before chemotherapy. Second, patients with gastrectomy lost the majority or the total of their stomach, which has a strong impact on nutrition absorption. All the researches that we have mentioned above focused on the GC patients in early stage who have underwent surgery with/without adjuvant chemotherapy. Therefore, the results may reflect the influence of both surgery and chemotherapy. Our study excluded all the patients with gastrectomy and focus on the influence of chemotherapy in the change of visceral fat and skeletal muscle. To the best of our knowledge, this is the first time that the composition parameters, visceral fat and skeletal muscle, are analyzed in MGC 
patients who lost the chance of the surgical resection at the initial diagnosis to predict prognosis and chemotherapy response.

Actually, compared with previous studies [15, $19,35,36]$, our study has several novelties. First, our study merely included metastatic gastric cancer who did not undergo gastrectomy, which exclude the influence of surgery in the content of visceral fat/skeletal muscle, which is different from previous studies. Second, our study established a new prognostic index, in which patients who had severe loss of visceral fat and skeletal muscle after chemotherapy had significantly shorter overall survival. Thus, we believe in patients who had severe loss of visceral fat and skeletal muscle after chemotherapy, the subsequent chemotherapy might not be suggested.

Our study also had some limitations. First, the sample size was small. Because of equipment replacement in our center, only one third of patients in the Phase II trial were enrolled in this study. Second, the CT scans were evaluated by only one experienced radiologist, which might have caused deviation. Third, we do not have a validation population to confirm our results. Actually, we have tried our best to search for an independent cohort to verify our results. However, the public database we have searched, including TCGA, SEER, ICGC, and GEO, have no information about abdominal fat content or skeletal muscle content. We have conducted a similar research in an ongoing phase III clinical trial in our center and try to verify our results.

In conclusion, VFA measured by CT scans can be used as a predictive factor for PFS and OS in MGC patients treated with EOF regimen. The severe loss of visceral fat and skeletal muscle can be used to predict shorter PFS and OS. In patients with severe loss of VFA and SMA after 2-cycle chemotherapy, the decision of subsequent chemotherapy should be made after deliberate consideration.

\section{Acknowledgements}

This study was funded by The National Key Research and Development Program of China (grant no. 2017YFC1308900).

\section{Author Contributions}

Wanjing Feng performed the statistical analysis and wrote the article. Xiaodong Zhu and Hui Zhu designed and managed the research. Mingzhu Huang, Xiaoying Zhao, Chenchen Wang, Jinjia Chang, Weijian Guo and Zhiyu Chen assisted with the research design.

\section{Ethics approval and consent to participant}

Informed consent was been obtained from all participants. The study was approved by the Ethics Committee of Fudan University Shanghai Cancer.

\section{Competing Interests}

The authors have declared that no competing interest exists.

\section{References}

1. Siegel RL, Miller KD, Jemal A. Cancer statistics, 2018. CA: a cancer journal for clinicians. 2018; 68: 7-30.

2. Hong JT, Kim TJ, Pyo JH, Kim ER, Hong SN, Kim YH, et al. Impact of sarcopenia on the risk of advanced colorectal neoplasia. Journal of gastroenterology and hepatology. 2019; 34: 162-8.

3. Bardou M, Barkun AN, Martel M. Obesity and colorectal cancer. Gut. 2013; 62: 933-47.

4. Duan XF, Tang P, Li Q, Yu ZT. Obesity, adipokines and hepatocellular carcinoma. International journal of cancer. 2013; 133: 1776-83.

5. Harimoto N, Shirabe K, Yamashita YI, Ikegami T, Yoshizumi T, Soejima Y, et al. Sarcopenia as a predictor of prognosis in patients following hepatectomy for hepatocellular carcinoma. The British journal of surgery. 2013; 100: 1523-30.

6. Vongsuvanh R, George J, Qiao L, van der Poorten D. Visceral adiposity in gastrointestinal and hepatic carcinogenesis. Cancer letters. 2013; 330: 1-10.

7. Donohoe CL, Doyle SL, Reynolds JV. Visceral adiposity, insulin resistance and cancer risk. Diabetology \& metabolic syndrome. 2011; 3: 12.

8. Aleksandrova K, Stelmach-Mardas M, Schlesinger S. Obesity and Liver Cancer. Recent results in cancer research Fortschritte der Krebsforschung Progres dans les recherches sur le cancer. 2016; 208: 177-98.

9. Donohoe CL, Pidgeon GP, Lysaght J, Reynolds JV. Obesity and gastrointestinal cancer. The British journal of surgery. 2010; 97: 628-42.

10. Goh LY, Goh KL. Obesity: an epidemiological perspective from Asia and its relationship to gastrointestinal and liver cancers. Journal of gastroenterology and hepatology. 2013; 28 Suppl 4: 54-8.

11. Ryall JG, Schertzer JD, Lynch GS. Cellular and molecular mechanisms underlying age-related skeletal muscle wasting and weakness. Biogerontology. 2008; 9: 213-28.

12. Seidell JC, Bakker CJ, van der Kooy K. Imaging techniques for measuring adipose-tissue distribution--a comparison between computed tomography and 1.5-T magnetic resonance. The American journal of clinical nutrition. 1990; 51: 953-7.

13. Yip C, Dinkel C, Mahajan A, Siddique M, Cook GJ, Goh V. Imaging body composition in cancer patients: visceral obesity, sarcopenia and sarcopenic obesity may impact on clinical outcome. Insights into imaging. 2015; 6: 489-97.

14. Blauwhoff-Buskermolen S, Versteeg KS, de van der Schueren MA, den Braver NR, Berkhof J, Langius JA, et al. Loss of Muscle Mass During Chemotherapy Is Predictive for Poor Survival of Patients With Metastatic Colorectal Cancer. Journal of clinical oncology : official journal of the American Society of Clinical Oncology. 2016; 34: 1339-44.

15. Li XT, Tang L, Chen Y, Li YL, Zhang XP, Sun YS. Visceral and subcutaneous fat as new independent predictive factors of survival in locally advanced gastric carcinoma patients treated with neo-adjuvant chemotherapy. Journal of cancer research and clinical oncology. 2015; 141: 1237-47.

16. Park SW, Lee HL, Doo EY, Lee KN, Jun DW, Lee OY, et al. Visceral Obesity Predicts Fewer Lymph Node Metastases and Better Overall Survival in Colon Cancer. Journal of gastrointestinal surgery : official journal of the Society for Surgery of the Alimentary Tract. 2015; 19: 1513-21.

17. Yamaoka Y, Fujitani K, Tsujinaka T, Yamamoto K, Hirao M, Sekimoto M. Skeletal muscle loss after total gastrectomy, exacerbated by adjuvant chemotherapy. Gastric cancer : official journal of the International Gastric Cancer Association and the Japanese Gastric Cancer Association. 2015; 18: $382-9$

18. Harada K, Baba Y, Ishimoto T, Kosumi K, Tokunaga R, Izumi D, et al. Low Visceral Fat Content is Associated with Poor Prognosis in a Database of 507 Upper Gastrointestinal Cancers. Annals of surgical oncology. 2015; 22: 3946-53.

19. Park HS, Kim HS, Beom SH, Rha SY, Chung HC, Kim JH, et al. Marked Loss of Muscle, Visceral Fat, or Subcutaneous Fat After Gastrectomy Predicts Poor Survival in Advanced Gastric Cancer: Single-Center Study from the CLASSIC Trial. Annals of surgical oncology. 2018; 25: 3222-30.

20. Zhu X, Zhao X, Peng W, Sun S, Cao J, Ji D, et al. Epirubicin combined with oxaliplatin and 5-day continuous infusion of 5-fluorouracil as a first-line treatment for metastatic gastric cancer: treatment outcomes and analysis of prognostic factors. Journal of cancer research and clinical oncology. 2015; 141: 109-18.

21. Fujiwara N, Nakagawa $H$, Kudo $Y$, Tateishi R, Taguri M, Watadani T, et al. Sarcopenia, intramuscular fat deposition, and visceral adiposity independently predict the outcomes of hepatocellular carcinoma. Journal of hepatology. 2015; 63: 131-40. 
22. Eisenhauer EA, Therasse P, Bogaerts J, Schwartz LH, Sargent D, Ford R, et al. New response evaluation criteria in solid tumours: revised RECIST guideline (version 1.1). European journal of cancer. 2009; 45: 228-47.

23. Shizgal HM. Body composition of patients with malnutrition and cancer. Summary of methods of assessment. Cancer. 1985; 55: 250-3.

24. Prado CM, Baracos VE, McCargar LJ, Mourtzakis M, Mulder KE, Reiman T, et al. Body composition as an independent determinant of 5-fluorouracil-based chemotherapy toxicity. Clinical cancer research : an official journal of the American Association for Cancer Research. 2007; 13: 3264-8.

25. Fielitz J. Cancer cachexia-when proteasomal inhibition is not enough. Journal of cachexia, sarcopenia and muscle. 2016; 7: 239-45.

26. Ongaro E, Buoro V, Cinausero M, Caccialanza R, Turri A, Fanotto V, et al. Sarcopenia in gastric cancer: when the loss costs too much. Gastric cancer : official journal of the International Gastric Cancer Association and the Japanese Gastric Cancer Association. 2017; 20: 563-72.

27. Fearon K, Strasser F, Anker SD, Bosaeus I, Bruera E, Fainsinger RL, et al. Definition and classification of cancer cachexia: an international consensus. The Lancet Oncology. 2011; 12: 489-95.

28. Skipworth RJ, Stewart GD, Dejong $\mathrm{CH}$, Preston $\mathrm{T}$, Fearon KC. Pathophysiology of cancer cachexia: much more than host-tumour interaction? Clinical nutrition. 2007; 26: 667-76.

29. Nam SY, Kim BC, Han KS, Ryu KH, Park BJ, Kim HB, et al. Abdominal visceral adipose tissue predicts risk of colorectal adenoma in both sexes. Clinical gastroenterology and hepatology : the official clinical practice journal of the American Gastroenterological Association. 2010; 8: 443-50 e1-2.

30. Kang HW, Kim D, Kim HJ, Kim CH, Kim YS, Park MJ, et al. Visceral obesity and insulin resistance as risk factors for colorectal adenoma: a cross-sectional, case-control study. The American journal of gastroenterology. 2010; 105: 178-87.

31. Yamaji T, Iwasaki M, Sasazuki S, Kurahashi N, Mutoh M, Yamamoto S, et al. Visceral fat volume and the prevalence of colorectal adenoma. American journal of epidemiology. 2009; 170: 1502-11.

32. Nagata N, Sakamoto K, Arai T, Niikura R, Shimbo T, Shinozaki M, et al. Visceral abdominal fat measured by computed tomography is associated with an increased risk of colorectal adenoma. International journal of cancer. 2014; 135: 2273-81.

33. Hu H, Cai Y, Huang J, Zhang J, Deng Y. Visceral adipose tissue and the risk of colorectal adenomas: a meta-analysis of observational studies. European journal of cancer prevention : the official journal of the European Cancer Prevention Organisation. 2015; 24: 462-9.

34. Balentine CJ, Enriquez J, Fisher W, Hodges S, Bansal V, Sansgiry S, et al. Intra-abdominal fat predicts survival in pancreatic cancer. Journal of gastrointestinal surgery : official journal of the Society for Surgery of the Alimentary Tract. 2010; 14: 1832-7.

35. Daly LE, Ni Bhuachalla EB, Power DG, Cushen SJ, James K, Ryan AM. Loss of skeletal muscle during systemic chemotherapy is prognostic of poor survival in patients with foregut cancer. Journal of cachexia, sarcopenia and muscle. 2018; 9: 315-25.

36. Lu J, Zheng ZF, Li P, Xie JW, Wang JB, Lin JX, et al. A Novel Preoperative Skeletal Muscle Measure as a Predictor of Postoperative Complications, Long-Term Survival and Tumor Recurrence for Patients with Gastric Cancer After Radical Gastrectomy. Annals of surgical oncology. 2018; 25: 439-48. 